\title{
ИСПОЛЬЗОВАНИЕ ГЕНЕТИЧЕСКИХ АЛГОРИТМОВ ДЛЯ ПОСТРОЕНИЯ ЭФФЕКТИВНЫХ КОМПЛЕКСНЫХ РАСПИСАНИЙ ОБРАБОТКИ ПАКЕТОВ ДАННЫХ В КОНВЕЙЕРНОЙ СИСТЕМЕ ПРИ ЗАДАНИИ ОГРАНИЧЕНИЙ НА ДЛИТЕЛЬНОСТЬ ИНТЕРВАЛОВ ВРЕМЕНИ ЕЕ ФУНКЦИОНИРОВАНИЯ
}

\author{
(C) 2020 К. В. Кротов \\ Севастопольский государственньй университет \\ ул. Университетская, 33, 299053 Севастополь, Российская Федерация
}

\begin{abstract}
Аннотация. В статье решается задача формирования эффективных комплексных расписаний обработки пакетов данных при задании ограничений на интервалы времени функционирования конвейерной системы. Задача формирования комплексных расписаний разделена на три подзадачи, каждая из которых решается на определенном уровне иерархии. На первом уровне определяются составы пакетов данных, на втором уровне определяются составы групп пакетов данных, которые обрабатывается в течение заданных интервалов времени функционирования системы, на третьем уровне определяется расписание обработки пакетов данных, входящих в каждую группу. Для решения задачи построения комплексных расписаний в составе такой иерархической системы применен аппарат теории иерархических игр. Рассматривается модель иерархической игры, позволяющая определять локально оптимальные решения на каждом уровне иерархии. Выполнено обоснование применения генетических алгоритмов для определения составов групп пакетов данных, которые являются эффективными с точки зрения минимизации простоев сегментов конвейера в течение заданных временных интервалов. Сформулирован способ кодирования решений по составам групп пакетов данных. Определены способы реализации генетических операторов, которые позволяют осуществить поиск локально оптимальных решений по составам групп пакетов на основе начального решения. Выполнена программная реализация процедур оптимизации составов пакетов данных, составов групп пакетов данных и расписаний обработки пакетов, входящих в группы. Исследования предложенного метода оптимизации, использующего генетические алгоритмы, показало, что его применение для формирования составов групп пакетов данных позволяет до 45 \% повысить эффективность использования ресурса времени сегментов конвейера по сравнению с начальным решением.

Ключевые слова: иерархическая игра, интервалы времени функционирования конвейерной системы, группы пакетов данных, генетические алгоритмы.
\end{abstract}

\section{ВВЕДЕНИЕ}

Развитием иерархического подхода по определению комплексных расписаний обработки пакетов данных (ПД), который предполагает формирование решений по их составам и расписаниям обработки [1], является задача построения комплексных расписаний

Кротов Кирилл Викторович e-mail: krotov_k1@mail.ru обработки ПД при задании ограничений на интервалы времени функционирования конвейерной системы. В этом случае обработка ПД в конвейерной системе выполняется в течение интервалов времени заданной длительности. Ограниченным ресурсом является время функционирования конвейерной системы, а составы пакетов и расписания их обработки должны быть сформированы так, чтобы выделяемый ограниченный ресурс был использован в максимальной степени. Контент доступен под лицензией Creative Commons Attribution 4.0 License. The content is available under Creative Commons Attribution 4.0 License. 
Для обработки ПД в течение заданных интервалов времени требуется сформировать их непересекающиеся множества, с каждым из которых выполняются операции в течение одного из интервалов. В результате должны быть сформированы непересекающиеся множества ПД, количество которых равно числу заданных интервалов времени. Тогда для решения задачи планирования обработки ПД в конвейерной системе требуется выполнить:

- формирование решений по составам пакетов;

- распределение сформированных ПД по их непересекающимся множествам, каждое из которых обрабатывается в течение одного из интервалов времени (формирование решений по составам множеств ПД, обрабатываемых в течение заданных временных интервалов);

- определение порядков выполнения операций на сегментах конвейера с пакетами, входящими в каждое из непересекающихся множеств.

Общая цель функционирования системы планирования, связанная с обработкой ПД в конвейерной системе в течение заданных интервалов времени, представлена в виде совокупности подцелей, достижение каждой из которых обеспечит формирование ПД, непересекающихся множеств пакетов, обрабатываемых в течение заданных временных интервалов, и расписаний обработки пакетов каждого из этих множеств. В соответствии с выполненной декомпозицией общей цели (функции) системы построения комплексных расписаний обработки ПД определены иерархически взаимосвязанные подфункции, каждая из которых выполняется на соответствующем уровне. На первом уровне определяется количество и составы ПД соответствующих типов, на втором уровне формируется совокупности пакетов, обрабатываемые в течение интервалов времени заданной длительности, на третьем уровне формируются расписания выполнения операций с ПД, назначенными для обработки в соответствующие временные интервалы. Совершенствование методов построения расписаний обработки ПД связано с применением теоретико-игрового подхода в теории расписаний [2].

\section{1. АНАЛИЗ СУЩЕСТВУЮЩИХ МЕТОДОВ ПОСТРОЕНИЯ КОМПЛЕКСНЫХ РАСПИСАНИЙ ОБРАБОТКИ ПАРТИЙ (ПАКЕТОВ) В КОНВЕЙЕРНЫХ СИСТЕМАХ}

Развитие современных методов построения расписаний обработки пакетов представлено в работах [3-12] (партий в понятиях указанных работ). В работах [3-6] рассматриваются методы решения задач формирования партий материалов, обрабатываемых непрерывными технологическим процессами (химическое производство). Под партиями в этом случае подразумевается объемы материалов, участвующих в процессе производства (объемы партий определяются способом производства требуемого вида продукта, количеством конечного продукта, определяемым в соответствии со спросом). В этих работах рассматриваются методы распределения обрабатываемых партий (размер которых не оптимизируется) материалов различных видов по параллельно действующим машинам. При этом рассматриваются задачи ограниченной размерности (2 продукта, 2-3 прибора в обрабатывающей системе). В работе [7] рассматривается решение задачи среднесрочного планирования выпуска продукции при заданном количестве обрабатывающих приборов и последующего составления расписаний обработки сформированных при планировании партий. При этом под партией подразумевается совокупность изделий одного типа, выпуск которых закрепляется за определенным предприятием или производственным участком. Размеры партий определяются в соответствии с заказами на производство и директивными сроками их выпуска. Модель оптимизации составов партий учитывает стоимостные параметры выпуска продукции, но не учитывает особенности технологических процессов. На основе полученного решения по распределению заказов по производственным участкам (сформированным составам партий) решается задача выделения для них ресурсов с целью реализации обработки. Совместно задача планирования (определения составов партий) и управления 
Генетический алгоритм построения комплексных расписаний обработки пакетов данных ...

выпуском (формирования расписаний обработки партий) в работе не решается. В работе [8] решается задача определения количества и составов партий единичных требований, обрабатываемых на одном приборе, с заданными директивными сроками обработки и стоимостью доставки партий. Составы партий разнотипных требований формируются с учетом директивных сроков. Тогда формирование партий предполагает и одновременное автоматическое построение расписания их обработки (вопрос оптимизации использования ресурса обрабатывающего прибора в данной работе не рассматривается). В результате решается только задача определения составов партий на основе заданных директивных сроков окончания их обработки. Аналогичная задача формирования партий требований в соответствии с их директивными сроками завершения, обрабатываемых на параллельных машинах, рассматривается в [9]. Объединение требований в партии реализуется с использованием эвристической процедуры в соответствии со значениями параметров начала обработки и директивными сроками окончания обработки. Так как обработка двух партий на одном приборе не может пересекаться, тогда сроки начала и окончания обработки партий используются для их распределения по параллельным машинам (расписание вытекает из сформированных составов партий). В работе [10] решается задача планирования производства полупроводников, предполагающая совместное формирование составов партий обрабатываемых пластин разных типов и расписаний обработки партий в конвейерной системе. Составы партий формируются, исходя из емкости печей обжига, которые являются обрабатывающими приборами. Планированию производственного процесса посвящена работа [11], в которой решается задача распределения заказов на производство продукции по сменным заданиям, распределения заданий по партиям и формирование расписаний обработки партий. Для определения составов сменных заданий используется эвристическая процедура, для определения составов партий разработана имитационная процедура, позволя- ющая моделировать прохождение партиями конвейерной системы. Определение эффективных составов партий предполагает задание параметров имитационной модели, соответствующих размерам партий изделий каждого типа, и проведение моделирования. В соответствии с результатами моделирования выбираются значения параметров размеров партий. В работе [12] реализуется решение задачи определения составов партий компонент, из которых выполняется формирование элементов (формирование комплектов из обработанных в системе изделий). Для формирования партий и расписаний их обработки введена оптимизационная модель, являющаяся многопараметрической и многоиндексной. Определение решений по количеству и составам партий осуществляется путем полного перебора возможных значений этих параметров.

Выполненный анализ существующих методов построения комплексных расписаний обработки ПД показал, что отсутствуют сформулированные алгоритмы планирования, учитывающие ограничения на интервалы времени функционирования конвейерной системы.

\section{2. МОДЕЛЬ ИЕРАРХИЧЕСКОЙ ИГРЫ ДЛЯ ОПРЕДЕЛЕНИЯ РАСПИСАНИЙ ОБРАБОТКИ ПД С УЧЕТОМ ОГРАНИЧЕНИЙ НА ВРЕМЯ ФУНКЦИОНИРОВАНИЯ КОНВЕЙЕРНОЙ СИСТЕМЫ}

Для обоснования модели иерархической игры, используемой для оптимизации составов ПД, составов групп ПД, расписаний обработки ПД, а также обоснования метода оптимизации групп ПД, введены следующие обозначения. Через $i$ обозначен идентификатор типа обрабатываемых в системе данных, $n-$ количество типов обрабатываемых в системе данных $(i=\overline{1, n}), n^{i}$ - количество данных $i$-го типа, которые должны быть обработаны в системе. Значения параметров $n^{i}>1(i=\overline{1, n})$. Тогда обрабатывающая данные $i$-го типа программа должна быть выполнена в конвейерной системе $n^{i}$ число раз, а для обработки од- 


\section{K. В. Кротов}

нотипных данных формируются ПД. Все сегменты конвейера характеризуются равными значениями производительности их работы, которые являются детерминированными и задаются перед реализацией планирования. Выполнение на $l$-м сегменте назначенной ему части $i$-й программы характеризуется длительностью обработки данных.

Обработка ПД разных типов выполняется в течение интервалов времени заданной длительности, обозначенных как $t^{z}$ (интервал времени, в течение которого реализуется обработка ПД $(z=\overline{1, Z}))$. На основе решения по количеству и составам ПД различных типов формируются решения по обработке пакетов в течение этих заданных интервалов $t^{z}$ $(z=\overline{1, Z})$. Тогда в рассмотрение введено понятие группы ПД. Группа ПД - это совокупность пакетов, обрабатываемых в течение одного интервала времени $t^{z}$ функционирования системы. Так как заданными являются ограничения на интервалы времени $t^{z}$ $(z=\overline{1, Z})$, тогда не все сформированные ПД могут быть включены в состав групп пакетов.

Количество обработанных в течение интервалов $t^{z}$ ПД зависит от их количества и составов (составы групп ПД зависят от количества и составов пакетов). Решение по порядку обработки ПД в каждой из групп является зависящим от составов пакетов в этой группе. Тогда определение количества и составов ПД, распределение ПД по группам, построение расписаний обработки ПД групп с учетом ограничений на длительность интервалов $t^{z}$ должно обеспечить обработку максимального количества данных.

В соответствии с выполненной декомпозицией обобщенной цели функционирования системы на совокупность иерархически упорядоченных целей подсистем, формирование решений осуществляется на уровнях системы следующим образом: первый уровень - peшения по составам ПД, второй - решения по составам групп ПД; третий - решения по порядку обработки на сегментах конвейера ПД, входящих в группы.

Для формирования решений по составам ПД $i$-х типов $(i=\overline{1, n})$ использованы обозначения [1]: $m_{i}-$ количество ПД $i$-го типа $(i=\overline{1, n})$, формируемых на первом уровне принятия решений, элементы $m_{i}$ образуют вектор $M ; A-$ матрица, элемент $a_{i h}$ которой это количество данных $i$-го типа в $h$-м ПД $\left(h \leq m_{i}\right)$. Формируемое решение имеет вид: $[M, A]$.

Для формирования решений по составам групп ПД введены обозначения: $N^{z}(z=\overline{1, Z})-$ группа ПД, обрабатываемых в течение одного из интервалов $t^{z}(z=\overline{1, Z}) ; m_{i}^{z}-$ количество ПД $i$-го типа в группе $N^{z} ;(A)_{i}^{z}-$ вектор количества данных $i$-го типа в $m_{i}^{z}$ ПД в группе $N^{z}$. Тогда ПД $i$-го типа, входящие в группу $N^{z}$, определены с использованием набора параметров вида: $\left[i, m_{i}^{z},(A)_{i}^{z}\right]$, а группа $N^{z}$ - это совокупность наборов в виде: $N^{z}=\left\{\left[i, m_{i}^{z},(A)_{i}^{z}\right]_{k} \mid\right.$ $\left.k=\overline{1, k_{z}}\right\}^{z}$, где $k_{z}$ - количество типов данных, пакеты которых входят в $N^{z}$. Вид решения, формируемого на втором уровне иерархии системы: $\left\{N^{z} \mid(z=\overline{1, Z})\right\}$.

В соответствии с решением по составу групп ПД требуется определить последовательности запуска ПД каждой из групп на обработку на сегментах конвейера, т. е. расписание обработки пакетов. Расписание обработки ПД группы $N^{z}$, обозначенное как $\pi^{z}$, представляет собой совокупность последовательностей $\pi^{l}$ запуска пакетов на обработку на $l$-х сегментах $(l=\overline{1, L})$. Расписание $\pi^{z}$ имеет вид: $\pi^{z}=\left\{\pi^{1}, \pi^{2}, . ., \pi^{L}\right\}^{z}$. Расписание $\pi^{z}$ для группы $N^{z}$ формируется в предположении, что порядок обработки ПД является одинаковым на всех сегментах конвейера. Для формализации вида последовательностей $\pi^{l}$ расписания $\pi^{z}$ в рассмотрении введена матрица порядка обработки $P^{z}$. Элемент $p_{i j}^{z}=1$, если ПД $i$-го типа занимает в последовательности $\pi^{l} j$-ю позицию, $p_{i j}^{z}=0$ в случае, если ПД $i$-го типа не занимает в последовательности $\pi^{l} j$-ю позицию. В рассмотрение введена матрица $R^{z}-$ матрица количества данных $i$-х типов в ПД, занимающих в $\pi^{l} j$-е позиции (элемент $r_{i j}^{z}$ равен количеству данных $i$-го типа в ПД, занимающем $j$-ю позицию в $\pi^{l}$, размерность матрицы $\left.k_{z} \times n_{p}^{z}\right)$. В дополнение к введенным в рассмотрение матрицам $P^{z}$ и $R^{z}$ вводится матрица $\left(t_{j q}^{0 l}\right)^{z}$, элементы $t_{j q}^{0 l}$ которой соответствуют моментам времени начала обработки $q$-х данных в пакете, занимающем $j$-ю пози- 
Генетический алгоритм построения комплексных расписаний обработки пакетов данных ...

цию в последовательности $\pi^{l}$ на $l$-м сегменте конвейера (для группы $N^{z}$ ). Решение, формируемое на третьем уровне иерархии системы, имеет вид: $\left.\left\{\left[P^{z},\left(t_{j q}^{0 l}\right)^{z} \mid(l=\overline{1, L})\right] \mid z=\overline{1, Z}\right)\right\}$.

В силу наличия ограничений на время функционирования системы не все сформированные ПД могут быть размещены в группах $N^{z}$. Для определения ПД, не вошедших в группы $N^{z}$, введено множество $Q$ вида: $Q=\left\{\left[i, m_{i}^{q},(A)_{i}^{q}\right]_{k} \mid k=\overline{1, k_{q}}\right\}$, где $m_{i}^{q}$ - количество ПД $i$-го типа, не вошедших в группы $N^{z}$, $(A)_{i}^{q}$ - вектор, каждый элемент которого представляет собой количество данных в каждом ПД $i$-го типа, не вошедших в группы $N^{z}$, $k_{q}$ - количество типов данных, пакеты которых не вошли в группы $N^{z}$.

Цель функционирования системы состоит в обработке максимального количества данных разных типов в течение заданных временных интервалов $t^{z}(z=\underline{1, Z})$. Тогда эффективное решение $\left\{N^{z^{*}} \mid(z=1, Z)\right\}$ обеспечивает реализацию этого требования для составов ПД. В соответствии с выполненной декомпозицией обобщенной функции системы на совокупность иерархически упорядоченных подфункций уровней, рассматриваемая задача построения расписаний групповой обработки ПД при наличии ограничений на длительность выполнения операций решается с использованием аппарата теории иерархических игр [2]. Следуя внешней цели функционирования системы, эффективность решений по составам ПД определяется количеством данных, обрабатываемым в группах (в течение интервалов $t^{z}$ ). Так как эффективные решения по составам ПД идентифицируются на первом уровне, тогда при определении значений критерия на этом уровне необходим учет решений по составам групп пакетов (со второго уровня иерархии). В силу того, что являются заданными ограничения на интервалы времени функционирования системы, поэтому требуется эффективное использование ее временного ресурса. Тогда при формировании решений по составам групп ПД необходим учет использования ресурса времени функционирования системы (при формировании групп ПД требуется использование ресурса времени функционирования систе- мы в максимальной степени). Анализ эффективности решений по составам групп ПД с точки зрения использования временного ресурса системы возможен с точки зрения формируемого на третьем уровне расписания обработки пакетов.

Таким образом, эффективность групп ПД на втором уровне оценивается на основе эффективных расписаний, составы групп ПД должны обеспечивать максимально эффективное использование временного ресурса сегментов конвейера. Эффективность составов ПД (первый уровень) оценивается на основе эффективных составов групп $N^{z}$, т. е. эффективный состав пакетов должен обеспечивать такой состав групп, при котором общее количество обработанных данных будет максимальным либо количество необработанных данных будет минимизировано. Критерии, формируемые для принятия решений на уровнях системы, должны учитывать: 1) третий уровень - эффективность использования оборудования конвейерной системы при размещении рассматриваемого ПД, добавляемого в расписание из соответствующей группы, в последовательностях $\pi^{l}(l=\overline{1, L})$; $2)$ второй уровень - общая эффективность использования ресурса времени конвейерной системы при обработке всех ПД во всех группах $N^{z}$; 3) первый уровень - общее количество данных, обработанных в системе в течение $Z$ интервалов времени.

С целью формирования модели оптимизации расписаний введены следующие обозначения: $\left(t_{i}^{l}\right)^{z}$ - вектор длительностей обработки данных $i$-х типов $l$-м сегменте конвейера $(l=\overline{1, L})$, пакеты которых включены в группу $N^{z}$ (всего определено $L$ векторов для каждой группы $N^{z}, z=\overline{1, Z}$ ). Выражения для расчета значений элементов матриц $\left(t_{j q}^{0 l}\right)^{z}$ аналогичны выражениям, введенным в рассмотрение в [1]. Использование выражений для $t_{j q}^{0 l}$ позволяет определить временные характеристики процесса выполнения конвейеризированных программ обработки ПД в каждой группе $N^{z}$ $(z=\overline{1, Z})$. Местоположение рассматриваемого пакета в $\pi^{l}$ может быть охарактеризовано текущими (для данного количества ПД в $\pi^{l}$ $(l=\overline{1, L}))$ простоями сегментов конвейера при 


\section{К. В. Кротов}

обработке пакетов, находящихся в последовательностях $\pi^{l}[1]$.

В [1] выполнено формирование вида критерия эффективности принятия решений при построении расписаний обработки ПД на нижнем уровне иерархии. С целью определения эффективных решений по порядкам обработки ПД в каждой из групп $N^{z}(z=\overline{1, Z})$ сформированный в [1] вид критерия модифицирован с учетом введенных обозначений, учитывающих особенности обработки пакетов в группах. Полученный после модификации критерий имеет следующий вид:

$$
\begin{gathered}
\sum_{l=2}^{L}\left(t_{11}^{0 l}\right)^{z}+ \\
+\sum_{l=1}^{L} \sum_{j=2}^{n_{p}^{z}}\left[\left(t_{j 1}^{0 l}\right)^{z}-\left[\left(t_{j-1, n}^{0 l}{ }_{j-1}\right)^{z}+\sum_{h=1}^{n^{z}}\left(t_{h}^{l}\right)^{z} \cdot p_{h, j-1}^{z}\right]\right]+ \\
+\sum_{l=2}^{L} \sum_{j=1}^{n_{p}^{z}} \sum_{q=2}^{n_{j}^{z}}\left[\left(t_{j q}^{0 l}\right)^{z}-\left[\left(t_{j, q-1}^{0 l}\right)^{z}+\sum_{h=1}^{n^{z}}\left(t_{h}^{l}\right)^{z} \cdot p_{h j}^{z}\right]\right]
\end{gathered}
$$

Первое слагаемое в (1) позволяет определить суммарные простои всех $L$ сегментов конвейера перед началом обработки данных в первой позиции $(q=1)$ в первом пакете $(j=1)$ в $\pi^{l}(l=\overline{1, L})$. Второе слагаемое определяет суммарные простои сегментов конвейера при переходе от обработки ПД в $(j-1)$-й позиции в $\pi^{l}$ к обработке ПД в $j$-й позиции в $\pi^{l}$. Третье слагаемое позволяет определить суммарные простои сегментов, вызванные ожиданием готовности данных при обработке их внутри ПД в группе $N^{z}(z=\overline{1, Z})$.

Критерий на втором уровне характеризует общую эффективность использования ресурса времени функционирования сегментов конвейера при реализации обработки ПД групп $N^{z}$. Критерий определяет общее время простоев сегментов конвейера при обработке ПД групп в течение заданных интервалов $t^{z}$ $(z=1, Z)$. Так как на втором уровне определяется состав групп ПД, то формируемый критерий характеризует эффективность решения $\left\{N^{z} \mid(z=1, Z)\right\}$ с точки зрения общих простоев сегментов в течение интервалов $t^{z}$ $(z=\overline{1, Z})$, которые определяются на основе расписаний, сформированных на третьем уровне для составов групп. Общее время простоев сегментов при обработке ПД группы $N^{z}$ определяется: 1) суммой длительностей интервалов наладки сегментов и возможного их простоя в ожидании начала обработки данных в первом пакете в $\pi^{l}(l=\overline{1, L})$ для $L$ сегментов; 2) суммой длительностей переналадки сегментов конвейера с обработки данных одного типа на обработку данных другого типа, возможного простоя сегментов в ожидании обработки данных в пакете в $j$-й позиции в $\pi^{l}$; 3) суммой длительностей интервалов времени простоев сегментов в ожидании готовности данных при обработке пакетов в последовательностях $\pi^{l}$ для всех $L$ сегментов; 4) суммой интервалов простоев $L$ сегментов после окончания обработки пакетов группы $N^{z}$ в количестве $n_{p}^{z}$. Первые три компоненты определяются соответствующими им слагаемыми в выражении (1) [1].

Для определения последней из компонент суммарного времени простоев использованы обозначения: $n_{p}^{z}-$ количество ПД, входящих в группу $N^{z}$ (индекс последнего ПД в группе); $n_{n_{p}^{z}}-$ количество данных, входящих в последний в $N^{z}$ ПД. Тогда $\left(t_{n_{p}^{z}, n_{n_{p}^{z}}^{0 l}}^{0 l}\right)^{z}-$ момент времени начала обработки последних данных в пакете с индексом $n_{p}^{z}$, время окончания обработки на $l$-м сегменте определяется выражением вида: $\left(t_{n_{p}^{z}, n_{n_{p}^{z}}^{0}}^{0 l}\right)^{z}+\sum_{h=1}^{n^{z}}\left(t_{h}^{l}\right)^{z} \cdot p_{h, n_{p}^{z}}^{z}$. Время простоя $l$-го сегмента после окончания обработки пакетов группы $N^{z}$ определяется выражением вида: $\left(t^{z}-\left[\left(t_{n_{p}^{z}, n_{p}^{z}}^{0 l}\right)^{z}++\sum_{h=1}^{n^{z}}\left(t_{h}^{l}\right)^{z} \cdot p_{h, n_{p}^{z}}^{z}\right]\right)$, а суммарные простои всех сегментов конвейера после окончания обработки группы ПД $N^{z}$ вычисляется с использованием выражения вида:

$$
\sum_{l=1}^{L}\left\{t^{z}-\left[\left(t_{n_{p}^{z} n_{p}^{z}}^{0 l}\right)^{z}+\sum_{h=1}^{n^{z}}\left(t_{h}^{l}\right)^{z} \cdot p_{h, n_{p}^{z}}^{z}\right]\right\} .
$$

С учетом выражений (1) и (2) определяется вид критерия эффективности решений по составам групп ПД $N^{z}(z=\overline{1, Z})$ на втором уровне в иерархической игре.

Решение, формируемое для групп ПД на втором уровне системы и передаваемое на первый уровень имеет вид: $\left\{N^{z^{*}} \mid z=\overline{1, Z}\right\}$, где $N^{z}=\left\{\left[i, m_{i}^{z},(A)_{i}^{z}\right]_{k} \mid k=\overline{1, k_{z}}\right\}^{z}$. Параметры $m_{i}^{z}$ и вектора $(A)_{i}^{z}$, входящие в $(k$-е) наборы па- 
Генетический алгоритм построения комплексных расписаний обработки пакетов данных ...

раметров группы $N^{z}$, обозначим соответственно $\left[m_{i}^{z}\right]_{k}$ и $\left[(A)_{i}^{z}\right]_{k}$. Количество данных в пакетах, входящих в $N^{z}$, определяется выражением вида: $\sum_{k=1}^{k_{z}} \sum_{h=1}^{\left[m_{i}^{z}\right]_{k}}\left[\left(a_{h}\right)_{i}^{z}\right]_{k}$, где $h$ - номер элемента в векторе $\left[(A)_{i}^{z}\right]_{k}$, а количество обрабатываемых данных, входящих во все группы $\left\{N^{z} \mid z=\overline{1, Z}\right\} \quad$ - выражением вида: $\sum_{z=1}^{Z} \sum_{k=1}^{k_{z}} \sum_{h=1}^{\left[m_{i}^{z}\right]_{k}}\left[\left(a_{h}\right)_{i}^{z}\right]_{k}$. Общее количество данных, которое должно быть обработано, определяется выражением вида: $\sum_{i=1}^{n} n^{i}$. Критерий эффективности решений на первом уровне, определяющий количество необработанных данных, имеет вид:

$$
\sum_{i=1}^{n} n^{i}-\sum_{z=1}^{Z} \sum_{k=1}^{k_{z}} \sum_{h=1}^{\left[m_{i}^{z}\right]_{k}}\left[\left(a_{h}\right)_{i}^{z}\right]_{k}
$$

Обобщая выражения (1)-(3), используемые при определении эффективных решений на соответствующих уровнях системы, представим модель иерархической игры для формирования ПД, групп ПД и расписаний обработки ПД в группах в следующем виде:

- первый уровень иерархии (определение составов ПД):

$\min f_{1}$, где

$$
f_{1}=\sum_{i=1}^{n} n^{i}-\sum_{z=1}^{Z} \sum_{k=1}^{k_{z}} \sum_{h=1}^{\left[m_{i}^{z}\right]_{k}}\left[\left(a_{h}\right)_{i}^{z}\right]_{k} ;
$$

- второй уровень иерархии (определение составов групп ПД $\left.N^{z}(z=\overline{1, Z})\right)$ :

$\min f_{2}$, где

$$
\begin{gathered}
f_{2}=\sum_{z=1}^{Z}\left[\sum_{l=2}^{L}\left(t_{11}^{0 l}\right)^{z}+\right. \\
\sum_{l=1}^{L} \sum_{j=2}^{n_{p}^{z}}\left[\left(t_{j 1}^{0 l}\right)^{z}-\left[\left(t_{j-1, n j-1}^{0 l}\right)^{z}+\sum_{h=1}^{k_{z}}\left(t_{h}^{l}\right)^{z} \cdot p_{h, j-1}^{z}\right]\right]+ \\
+\sum_{l=2}^{L} \sum_{j=1}^{n_{p}^{z}} \sum_{q=2}^{n_{j}^{z}}\left[\left(t_{j q}^{0 l}\right)^{z}-\left[\left(t_{j, q-1}^{0 l}\right)^{z}+\sum_{h=1}^{k_{z}}\left(t_{h}^{l}\right)^{z} \cdot p_{h j}^{z}\right]\right]+ \\
+\left[\sum_{l=1}^{L}\left(t^{z}-\left[\left(t_{n_{p}^{z}, n_{n p}^{z}}^{0 l}\right)^{z}+\sum_{h=1}^{k_{z}}\left(t_{h}^{l}\right)^{z} \cdot p_{h, n_{p}^{z}}^{z}\right]\right] ;\right.
\end{gathered}
$$

- третий уровень иерархии (определение порядков обработки ПД в группах $N^{z}$ - расписаний обработки ПД в конвейерной системе):

$$
\min f_{3}^{z} \text {, где }
$$

$$
\begin{gathered}
f_{3}^{z}=\sum_{l=2}^{L}\left(t_{11}^{0 l}\right)^{z}+ \\
+\sum_{l=1}^{L} \sum_{j=2}^{n_{p}^{z}}\left[\left(t_{j 1}^{0 l}\right)^{z}-\left[\left(t_{j-1, n}^{0 l}{ }_{j-1}\right)^{z}+\sum_{h=1}^{k_{z}}\left(t_{h}^{l}\right)^{z} \cdot p_{h, j-1}^{z}\right]\right]+(6) \\
+\sum_{l=2}^{L} \sum_{j=1}^{n_{p}^{z}} \sum_{q=2}^{n_{j}^{z}}\left[\left(t_{j q}^{0 l}\right)^{z}-\left[\left(t_{j, q-1}^{0 l}\right)^{z}+\sum_{h=1}^{k_{z}}\left(t_{h}^{l}\right)^{z} \cdot p_{h j}^{z}\right]\right], \\
\text { при } z=\overline{1, Z} \text {; } \\
\text { - ограничение на третьем уровне иерар- }
\end{gathered}
$$
хии для длительности реализации расписаний обработки ПД группы $N^{z}$ :

$$
\begin{aligned}
\max _{l}\left[\left(t_{n_{p}^{z}, n_{n_{p}^{z}}^{2}}^{0 l}\right)^{z}+\sum_{h=1}^{k_{z}}\left(t_{h}^{l}\right)^{z} \cdot p_{h, n_{p}^{z}}^{z}\right] \leq t^{z}, \\
\text { при } z=\overline{1, Z .}
\end{aligned}
$$

Введенная в рассмотрение модель (4)-(7) используется для определения решений по комплексным расписаниям обработки ПД в конвейерной системе с учетом ограничений на длительности интервалов времени ее функционирования. Для формирования решений на соответствующих уровнях системы комплексного планирования обработки ПД требуется разработка соответствующих методов оптимизации (в частности, метода оптимизации составов групп ПД, обрабатываемых в течение заданных интервалов времени $\left.t^{z}(z=\overline{1, Z})\right)$.

\section{3. МЕТОД ФОРМИРОВАНИЯ НАЧАЛЬНОГО РЕШЕНИЯ ПО СОСТАВАМ ГРУПП ПД, ОБРАБАТЫВАЕМЫХ В ТЕЧЕНИЕ ИНТЕРВАЛОВ ВРЕМЕНИ ФУНКЦИОНИРОВАНИЯ СИСТЕМЫ}

Метод построения эффективных составов групп $N^{z}$ предполагает выполнение двух этапов: 1) формирование начального решения (начального состава групп ПД); 2) переход к локально оптимальному решению по составам групп $\left\{N^{z^{*}} \mid z=\overline{1, Z}\right\}$. Формирование начального решения возможно путем реализации двух подходов: 1) с использованием формулируемого детерминированного алгоритма, который последовательно распределяет ПД по группам $N^{z}$ с учетом ограничений на длительность интервалов функционирования 


\section{К. В. Кротов}

системы; 2) использование стохастического способа построения решений по составам групп ПД на основе генетических алгоритмов (ГА). Рассмотрим детерминированный алгоритм формирования начальных решений по составам групп ПД, который реализует распределение ПД, полученных в решении $[M, A][1]$ с первого уровня иерархии системы, по множествам наборов параметров $N^{z}=\left\{\left[i, m_{i}^{z},(A)_{i}^{z}\right]_{k}, \mid k=\overline{1, k_{z}}\right\}^{z}$ и множеству $Q$ с учетом ограничений на время обработки пакетов в группах.

Рассмотрение алгоритма построения начального решения $\left\{N^{z} \mid z=\overline{1, Z}\right\}$ предваряется введением обозначений: 1) $i^{\prime}-$ тип данных, пакет которых размещается в группе $N^{z}$; 2) $m_{i^{\prime}}-i^{\prime}$-й элемент вектора $M$, соответствующий количеству ПД $i^{\prime}$-го типа, размещаемых в группах $N^{z}(z=\overline{1, Z})$ и множестве $Q$; 3) $z^{\prime}-$ индекс текущей рассматриваемой группы, в которую добавляется ПД $i^{\prime}$-го типа; 4) $h^{i^{\prime}}$ - номер ПД $i^{\prime}$-го типа, размещаемого в группе $N^{z^{\prime}}$, число данных в котором - это значение элемента $a_{i^{\prime} h^{i}}$ матрицы $A\left(h^{i^{\prime}}=\overline{1, m_{i^{\prime}}}\right)$; 6) $I_{1}^{z}$ - множество типов данных, пакеты которых размещаются в группах $N^{z}(z=\overline{1, Z})$; 7) $I_{2}^{z}$ - изменяемое в процессе реализации алгоритма множество типов данных, пакеты которых размещаются в группах $N^{z}(z=\overline{1, Z})$; 9) $N Z$ - множество номеров групп $N^{z}$, в которых размещаются ПД; 10) $N Z_{T}$ - изменяемое в процессе реализации алгоритма множество номеров групп $N^{z}$.

С целью учета ограничений на длительности интервалов времени функционирования системы в рассмотрение введены условия включения ПД в рассматриваемую группу и исключения группы из дальнейшего рассмотрения в процессе формирования начального решения. В соответствии с [1] момент времени окончания обработки последнего в $\pi^{l}$ ПД (с индексом $n_{p}^{z}$ ) определяется выражением: $\left(t_{n_{p}^{z}, n_{n_{p}^{z}}^{0 l}}^{z}\right)^{z}+\sum_{j=1}^{k_{z}}\left(t_{j}^{l}\right)^{z} \cdot p_{j, n_{p}^{z}}^{z}$. Тогда условие включения текущего рассматриваемого ПД в группу $N^{z}$, имеет вид:

$$
\max _{l}\left[\left(t_{n_{p}^{z}, n_{n_{p}^{z}}}^{0 l}\right)^{z}+\sum_{j=1}^{k_{z}}\left(t_{j}^{l}\right)^{z} \cdot p_{j, n_{p}^{z}}^{z}\right] \leq t^{z},
$$

а условие исключения группы из дальнейшего рассмотрения при формировании начального решения (так как она является полностью заполненной ПД) имеет вид:

$$
t^{z}-\max _{l}\left[\left(t_{n_{p}^{z}, n_{n_{p}^{z}}}^{0 l}\right)^{z}+\sum_{j=1}^{k_{z}}\left(t_{j}^{l}\right)^{z} \cdot p_{j, n_{p}^{z}}^{z}\right]=0 .
$$

Начальная инициализация параметров для реализации алгоритма формирования начального состава групп ПД выполнена следующим образом: 1) $i=1$ (формирование групп $N^{z}$ выполняется, начиная с данных $(i=1)$-го типа); 2) $k_{z}=0(z=\overline{1, Z})$; 3) $k_{q}=0$; 4) инициализация множеств наборов параметров $N^{z}$ $(z=\overline{1, Z})$ и множества $Q$ в виде: $N^{z}=\varnothing ; Q=\varnothing$ (наборы вида $\left[i, m_{i}^{z},(A)_{i}^{z}\right]$ и $\left[i, m_{i}^{q},(A)_{i}^{q}\right]$ будут добавляться в состав соответствующих множеств); 6) $I_{1}^{z}=\{1,2, . ., n\}$; $\left.\quad 7\right) \quad I_{2}^{z}=I_{1}^{z}$; 8) $N Z=\{1,2, . ., Z\}$; 9) $h^{i}=1 \quad(i=\overline{1, n})$, т. е. для каждого $i$-го типа задан идентификатор ПД, размещаемого в группах $N^{z}(z=1, Z)$.

Алгоритм формирования начального решения по составам групп ПД $\left\{N^{z} \mid z \in N Z\right\}$ содержит следующие шаги:

1) инициализация состава множества $N Z_{T}$ номеров групп, в которых на данной итерации алгоритма будут размещаться ПД: $N Z_{T}=N Z$;

2) инициализация номера текущей рассматриваемой группы $N^{z}$, в которой будут размещаться ПД $i^{\prime}$-го типа: $z^{\prime}=\min \left\{z \mid z \in N Z_{T}\right\}$, $N Z_{T}=N Z_{T} \backslash\left\{z^{\prime}\right\}$

3) определение типа данных $i^{\prime}$, пакет которых размещается в группах $N^{z}\left(z \in N Z_{T}\right)$ : $i^{\prime}=\min \left\{i \mid i \in I_{2}^{z}\right\}, I_{2}^{z}=I_{2}^{z} \backslash\left\{i^{\prime}\right\}$;

4) для $i^{\prime}$-го типа данных и группы $N^{z^{\prime}}$ выполняется проверка условия $\left[i^{\prime}, m_{i^{\prime}}^{z^{\prime}},(A)_{i^{\prime}}^{z^{\prime}}\right] \in N^{z^{\prime}}$; если условие выполняется, то переход на шаг 6;

5) реализуется формирование набора параметров вида $\left[i^{\prime}, m_{i^{\prime}}^{z^{\prime}},(A)_{i^{\prime}}^{z^{\prime}}\right]$; начальная инициализация значений параметров набора в виде: $m_{i^{\prime}}^{z^{\prime}}=0$, сформированный набор параметров $\left[i, m_{i^{\prime}}^{z^{\prime}},(A)_{i^{\prime}}^{z^{\prime}}\right]$ включается в состав группы ПД $N^{z^{\prime}}: k_{z^{\prime}}=k_{z^{\prime}}+1 ; k=k_{z^{\prime}}, \quad N^{z^{\prime}}=N^{z^{\prime}} \cup$ $\cup\left\{\left[i^{\prime}, m_{i^{\prime}}^{z^{\prime}},(A)_{i^{\prime}}^{z^{\prime}}\right]_{k}\right\}$;

6) на основе полученного с первого уровня решения $[M, A]$ для $i^{\prime}$-го типа данных инициализируются значения параметров набора $\left[i^{\prime}, m_{i^{\prime}}^{z^{\prime}},(A)_{i^{\prime}}^{z^{\prime}}\right]_{k} \in N^{z^{\prime}}: m_{i^{\prime}}^{z^{\prime}}=m_{i^{\prime}}^{z^{\prime}}+1 ;\left(a_{m_{i}^{z}}\right)_{i}^{z^{\prime}}=a_{i^{\prime} h^{\prime}}-$ реализуется формирование решения по составу группы ПД $N^{z^{\prime}}$; 
Генетический алгоритм построения комплексных расписаний обработки пакетов данных ...

7) сформированное решение по составу группы ПД $N^{z^{\prime}}$ передается на третий уровень иерархии для формирования эффективного расписания обработки пакетов $\pi^{z^{\prime}}$;

8) получение с третьего уровня иерархии расписания $\pi^{z^{\prime}}$ в виде решения $\left[P^{z^{\prime}},\left\{\left(t_{j q}^{0 l}\right)^{z^{\prime}}\right.\right.$ $l=\overline{1, L}\}]$; проверка для расписания $\pi^{z^{\prime}}$ выполнения ограничения вида (8) на длительность его реализации; если ограничение вида (8) выполняется, то $h^{i^{\prime}}=h^{i^{\prime}}+1$, реализуется переход на шаг 9; если условие (8) не выполняется, то реализуется переход на шаг 13 (исключение ПД і'-го типа из состава группы $N^{z^{\prime}}$ );

9) проверка для сформированного состава группы $N^{z^{\prime}}$ (в которую добавлен пакет с количеством данных, равным $\left.\left(a_{m_{z^{\prime}}^{\prime}}\right)_{i^{\prime}}^{z^{\prime}}\right)$ выполнения условия (9);

10) если ограничение на длительность реализации расписания вида (8) выполнено, условие вида (9) не выполняется, то осуществляется переход к шагу 12;

11 ) если условие (9) выполнено (автоматическивыполненоусловие (8)), то $N Z=N Z \backslash\left\{z^{\prime}\right\}$ (ПД в группе $N^{z^{\prime}}$ не могут быть размещены);

12) проверка условия $h^{i^{\prime}}>m_{i^{\prime}}$, при его истинности все ПД $i^{\prime}$-го типа размещены в группах $N^{z}(z \in N Z)$ и множестве $Q$, тогда множество типов данных $I_{1}^{z}$, ПД которых размещаются в группах $N^{z}$, модифицируется: $I_{1}^{z}=I_{1}^{z} \backslash\left\{i^{\prime}\right\}$; реализуется переход на шаг 20; если условие $h^{i^{\prime}}>m_{i^{\prime}}$ не выполняется, то реализуется переход на шаг 20;

13) модификация сформированного решения по составу группы $N^{z^{\prime}}$ следующим образом: $\left(a_{m_{i}^{z_{i}^{\prime}}}\right)_{i^{\prime}}^{z^{\prime}}=0 ; m_{i^{\prime}}^{z^{\prime}}=m_{i^{\prime}}^{z^{\prime}}-1$ (ПД $i^{\prime}$-го типа с количеством элементов $\left(a_{m_{i}^{z^{\prime}}}\right)_{i^{\prime}}^{z^{\prime}}$ исключается из группы $N^{z^{\prime}}$ );

14) если получено $m_{i^{\prime}}^{z^{\prime}}=0, \quad$ то $N^{z^{\prime}}=N^{z^{\prime}} \backslash\left\{\left[i^{\prime}, m_{i^{\prime}}^{z^{\prime}},(A)_{i^{\prime}}^{z^{\prime}}\right]_{k}\right\}, k_{z^{\prime}}=k_{z^{\prime}}-1$ (из группы $N^{z^{\prime}}$ исключается набор $\left[i^{\prime}, m_{i^{\prime}}^{z^{\prime}},(A)_{i^{\prime}}^{z^{\prime}}\right]_{k}$, ПД $i^{\prime}$-го типа в группу $N^{z^{\prime}}$ не включены);

15) если $N Z_{T} \neq \varnothing$, то определяется номер следующей группы $N^{z^{\prime}}$, в которую может быть включен ПД $i^{\prime}$-го типа: $z^{\prime}=\min \left\{z \mid z \in N Z_{T}\right\}$, модификация множества $N Z_{T}: N Z_{T}=N Z_{T} \backslash\left\{z^{\prime}\right\}$; реализуется переход к шагу 4 ;

16) если $N Z_{T}=\varnothing$, то рассматриваемый ПД $i^{\prime}$-го типа (количество данных $a_{i^{\prime} h^{\prime}}$ ) ни в одну из групп $N^{z}(z \in N Z)$ размещен быть не может, тогда выполняется проверка условия $\left[i^{\prime}, m_{i^{\prime}}^{q},(A)_{i^{\prime}}^{q}\right] \in Q$ для этого $\left(i^{\prime}\right.$-го) типа данных;

17) привыполненииусловия $\left[i^{\prime}, m_{i^{\prime}}^{q},(A)_{i^{\prime}}^{q}\right] \in Q$ реализуется модификация значений параметров $i^{\prime}, \quad m_{i^{\prime}}^{q}, \quad(A)_{i^{\prime}}^{q}$ следующим образом: $m_{i^{\prime}}^{q}=m_{i^{\prime}}^{q}+1,\left(a_{m_{i}^{q}}\right)_{i^{\prime}}=a_{i^{\prime} h^{\prime}}$; модификация номера рассматриваемого ПД $i^{\prime}$-го типа $\left(h^{i^{\prime}}=h^{i^{\prime}}+1\right)$; переход на шаг 19;

18) если $\left[i^{\prime}, m_{i^{\prime}}^{q},(A)_{i^{\prime}}^{q}\right] \notin Q$, то формируется набор параметров вида $\left[i^{\prime}, m_{i^{\prime}}^{q},(A)_{i^{\prime}}^{q}\right]$, который включается в множество $Q: k_{q}=k_{q}+1 ; k=k_{q}$; $Q=Q \cup\left\{\left[i^{\prime}, m_{i^{\prime}}^{q},(A)_{i^{\prime}}^{q}\right]_{k}\right\}$; выполняется инициализация значений параметров: $m_{i^{\prime}}^{q}=1$, $\left(a_{m_{i}^{q}}\right)_{i^{\prime}}=a_{i^{\prime} h^{i}}$; модификация номера ПД $i^{\prime}$-го типа $\left(h^{i^{\prime}}=h^{i^{\prime}}+1\right)$;

19) проверка условия $h^{i^{\prime}}>m_{i^{\prime}}$ при его истинности все ПД $i^{\prime}$-го типа размещены в группах $N^{z}(z \in N Z)$ и множестве $Q$, тогда множество типов данных $I_{1}^{z}$, пакеты которых размещаются в группах $N^{2}$, модифицируется: $I_{1}^{z}=I_{1}^{z} \backslash\left\{i^{\prime}\right\}$, выполняется переход к шаг 20;

20) проверка выполнения условия $I_{2}^{z} \neq \varnothing$, в случае истинности условия реализуется инициализация множества $N Z_{T}$ идентификаторов групп, в которых могут быть размещены ПД, следующим образом: $N Z_{T}=N Z$, выполняется переход на шаг 3;

21) при истинности условия $I_{2}^{z}=\varnothing$ по одному ПД каждого $i$-го типа $\left(i \in I_{1}^{z}\right)$ размещено в группах $N^{z}(z \in N Z)$, при $I_{1}^{z} \neq \varnothing$ имеются типы данных, пакеты которых не размещены в группах $N^{z}(z \in N Z)$, тогда выполняется инициализация множеств $I_{2}^{z}$ и $N Z_{T}$ следующим образом: $I_{2}^{z}=I_{1}^{z} ; N Z_{T}=N Z$; реализуется переход на шаг 3 ; при $I_{1}^{z}=\varnothing$ подучено начальное решение по составам групп $N^{z}$ $(z=\overline{1, Z})$, переход на шаг 22;

22) полученные составы групп $N^{z}(z=\overline{1, Z})$ интерпретируются как локально оптимальное решение: $\left\{N^{z^{*}} \mid z=\overline{1, Z}\right\}=\left\{N^{z} \mid z=\overline{1, Z}\right\}$; останов алгоритма.

Реализация рассмотренного алгоритма позволяет сформировать начальное решение по составам групп ПД, которое требуется оптимизировать с точки зрения минимизации суммарных простоев сегментов конвейера при обработки пакетов в группах. Для этого на основе решения вида $\left\{N^{z} \mid z=\overline{1, Z}\right\}$ форми- 


\section{К. В. Кротов}

руется начальная популяция хромосом, используемая для дальнейшей оптимизации решений.

\section{4. МЕТОД ОПРЕДЕЛЕНИЯ ЭФФЕКТИВНЫХ РЕШЕНИЙ ПО СОСТАВАМ ГРУПП ПАКЕТОВ ДАННЫХ, ИСПОЛЬЗУЮЩИЙ ГЕНЕТИЧЕСКИЕ АЛГОРИТМЫ}

Формирование эффективных составов групп $N^{z}(z=\overline{1, Z})$ на основе начального решения $\left\{N^{z} \mid z=\overline{1, Z}\right\}$ предполагает определение таких их составов, при которых суммарное время простоев сегментов конвейера при обработке ПД в группах $N^{z}(z=\overline{1, Z})$ будет минимальным. Решение задачи оптимизации составов групп ПД реализуется посредством использования генетических алгоритмов (ГА). Стандартными компонентами ГА являются: способ кодирования решений, способы формирования новых решений, предусматривающие «скрещивание» имеющихся решений (кроссинговер) и мутацию генов рассматриваемых хромосом (формирование новых значений генов), а также способ выбора решений в популяции, на основе которых будут формироваться новые решения (селекция). Поэтому применение генетических алгоритмов к решению рассматриваемой задачи предполагает обоснование способа кодирования решений и способов реализации операторов скрещивания, мутации и селекции.

Исходное решение по составам ПД имеет вид $[M, A]$. Тогда по группам должны быть распределены ПД первого типа в количестве $m_{1}$, ПД второго типа в количестве $m_{2}$ и т. д. ПД $n$-го типа в количестве $m_{n}$. При сопостав- лении гену в хромосоме одного ПД размер хромосомы определяется как $\sum_{i=1}^{n} m_{i}$. Для каждого $i$-го типа данных $(i=\overline{1, n})$ в хромосоме выделяется участок из $m_{i}$ генов. Каждому $h_{i}$-му гену в участке хромосомы для $i$-го типа данных соответствует ПД, имеющий номер $h_{i}$ и принадлежащий одной из групп $N^{z}(z=\overline{1, Z})$ или множеству $Q$. Составы ПД некоторого $i$-го типа, которые сопоставляются с $h_{i}$-ми генами, входящими в $i$-й участок хромосомы, определяются значениями элементов $\left(a_{h_{i}}\right)_{i}^{z}$ в матрицах $(A)_{i}^{z}$, принадлежащих наборам параметров вида $\left[i, m_{i}^{z},(A)_{i}^{z}\right]_{k}$, которые входят в разные группы $N^{z}(z=\overline{1, Z})$. Значение гена $q_{h_{i}}$ в участке хромосомы, сопоставленным с ПД $i$-го типа, равно либо номеру группы $N^{z}$ $(z=\overline{1, Z})$, в которую входит пакет этого типа с соответствующим $h_{i}$-м номером, либо значению 0 , если пакет входит в множество $Q$. Пакеты данных $i$-го типа из групп $N^{z}(z=\overline{1, Z})$ последовательно «распределяются» по генам участка хромосомы, который соответствует данным этого типа. Пример реализации способа кодирования решений для случая $n=3$, $m_{i}=8(i=\overline{1,3}), Z=3$ представлен на рис. 1 .

На основе каждого решения $\left\{N^{z} \mid z=\overline{1, Z}\right\}$ формируется хромосома соответствующего вида. После реализации генетических операторов, обеспечивающих формирование новой популяции хромосом, на основе каждой хромосомы из полученного их набора вновь формируется решение по составу групп ПД вида $\left\{N^{z} \mid z=\overline{1, Z}\right\}$, которое передается на третий уровень для построения на его основе расписаний обработки ПД, входящих в каждую из групп. После построения расписа-

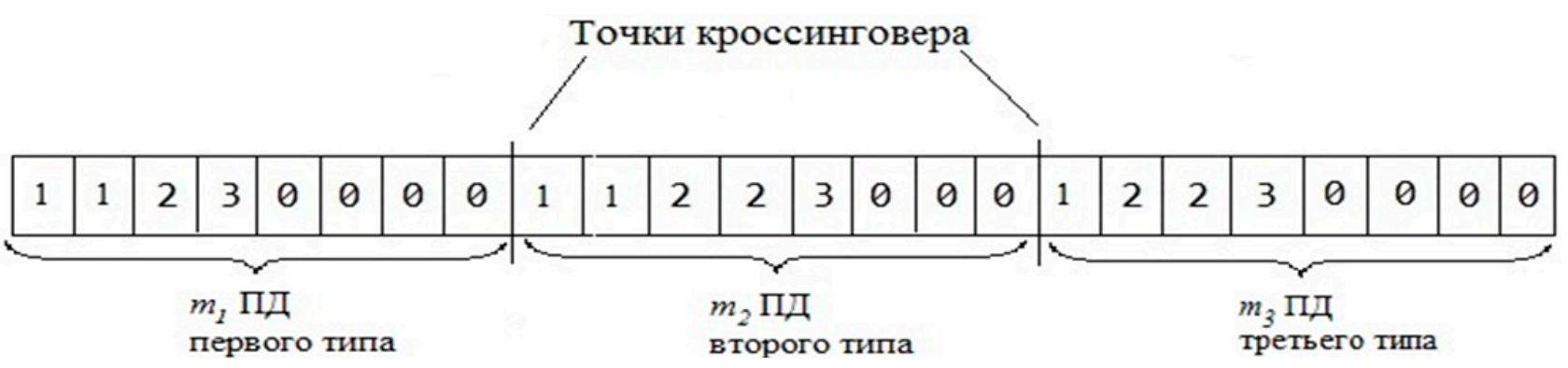

Рис. 1. Вид хромосомы, соответствующей решению $\left\{N^{z} \mid z=\overline{1, Z}\right\}$ по составу групп ПД [Fig. 1. View of the chromosome corresponding to the decision on the composition of groups of data packets] 
Генетический алгоритм построения комплексных расписаний обработки пакетов данных ...

ния, соответствующего ПД группы $N^{z}$, реализуется проверка выполнения ограничения вида (8). В случае его выполнения (при учете сформированного расписания для ПД группы) решение по составу этой группы анализируется с точки зрения его эффективности с использованием выражения (5).

Формирование новых решений по составам групп ПД в виде хромосом (новой популяции хромосом) осуществляется путем реализации операторов кроссинговера и мутации с последующей селекцией полученных решений с точки зрения введенного критерия. Оператор мутации предполагает:

- генерацию для каждого участка хромосомы, соответствующего определенному типу данных, номера гена $h_{i}$, мутация которого будет выполняться (формируется n точек мутации в случае, если для каждого участка хромосомы определяется одна точка мутации, и $k n$ точек мутации, если для каждого участка хромосомы формируется $k$ точек);

- определение (стохастическим образом) вида операции, связанной с перемещением рассматриваемого ПД с идентификатором $h_{i}$ между группами $N^{z}(z=\overline{1, Z})-$ определяется либо увеличение значения $q_{h_{i}}$ этого гена (перемещение ПД с идентификатором $h_{i}$ в группу $N^{z}$ с большим номером - единичное значение генерируемой случайным образом переменной), либо уменьшение значения $q_{h_{i}}$ этого гена (перемещение ПД с идентификатором $h_{i}$ в группу $N^{z}$ с меньшим номером нулевое значение генерируемой случайным образом переменной);

В том случае, если полученное после мутации значение гена удовлетворяет условию $q_{h_{i}}>Z$, то это значение инициализируется следующим образом: $q_{h_{i}}=0$. Если формируемый перед реализацией мутации случайным образом идентификатор $h_{i}$ соответствует гену, значение которого $q_{h_{i}}$ первоначально равно 0, и формируется тип операции, реализующей уменьшение значения гена (что является невозможным), то номер гена для рассматриваемого $i$-го типа данных генерируется повторно.

Оператор кроссинговера предполагает генерацию одной либо нескольких точек пере- сечения участков хромосомы, каждый из которых соответствует определенному $i$-му типу данных. В результате путем пересечения участков родительских хромосом формируются хромосомы в новой популяции.

Для реализации селекции полученные после реализации операторов мутации и кроссинговера хромосомы преобразуются в стандартную форму решений для групп ПД вида

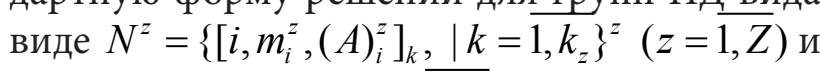
$Q=\left\{\left[i, m_{i}^{q},(A)_{i}^{q}\right]_{k}, \mid k=\overline{1, k_{q}}\right\}$. Полученные составы групп $N^{z}(z=\overline{1, Z})$ передаются на третий уровень иерархии для формирования расписаний обработки ПД каждой из групп. Полученные расписания в виде $\left\{\left[P^{z},\left(t_{j q}^{0 l}\right)^{z} \mid\right.\right.$ $(l=\overline{1, L})] \mid z=\overline{1, Z})\}$ для каждой группы ПД интерпретируются с точки зрения выполнения условия (8). При истинности ограничения (8) рассматриваемое решение интерпретируется (включается в формируемую популяцию), при ложности этого условия полученное решение (хромосома) отбрасывается (не интерпретируется).

После формирования совокупности допустимых решений по составам групп ПД выполняется их сортировка по значению критерия $f_{2}$. Затем заданное количество решений из рассматриваемой совокупности формируют новую популяцию, с которой в дальнейшем выполняются преобразования.

\section{5. ОБСУЖДЕНИЕ РЕЗУЛЬТАТОВ ИССЛЕДОВАНИЙ МЕТОДА ОПРЕДЕЛЕНИЯ ЭФФЕКТИВНЫХ СОСТАВОВ ГРУПП ПД}

Для исследования эффективности применения метода оптимизации составов групп ПД использованы обозначения: $\max \left(t_{i}^{l}\right) / \min \left(t_{i}^{l}\right)-$ отношение максимальной длительности обработки данных $i$-х типов $(i=\overline{1, n})$ на $l$-х сегментах конвейера $(l=\overline{1, L})$ к минимальной длительности обработки данных этих типов; $\max \left(t_{i j}^{l}\right) / \min \left(t_{i j}^{l}\right)$ - отношение максимальной длительности переналадки сегментов конвейера $(l=\overline{1, L})$ к минимальной длительности переналадки сегментов. Задаваемые значения параметров $n=5, L=5, n^{i}=24$. Значе- 


\section{К. В. Кротов}

ния параметра $t^{z}$ задавались равными 100 и 200 , значения параметра $Z-$ соответственно 2 и 4 . Значения отношения $\max \left(t_{i j}^{l}\right) / \min \left(t_{i j}^{l}\right)$ задавались равными: $1,2,4,8,16$, а отношения $\max \left(t_{i}^{l}\right) / \min \left(t_{i}^{l}\right)$ - равными $1,2,4,8$. При исследованиях эффективности метода определения групп ПД формировались следующие решения:

- фиксированные группы ПД (решения $[M, A]$ по составам ПД использовались для определения начальных решений по составам групп $N^{z}(z=\overline{1, Z})$ без оптимизации);

- группы ПД, оптимизированные с использованием рассмотренного генетического алгоритма.

При исследовании эффективности метода формирования групп ПД на первом и третьем уровнях использованы методы оптимизации решений, рассмотренные в [1]. Параметром эффективности применения метода формирования групп ПД является отношение разности значений общих простоев сегментов конвейера при формировании фиксированных групп и групп, составы которых сформированы в соответствии с рассмотренным методом, к значению длительности общих простоев сегментов конвейеров для фиксированных групп $N^{z}(z=\overline{1, Z})$. Графики зависимостей эффективности применения метода оптимизации групп ПД от значений входных параметров представлены на рис. 2-5.

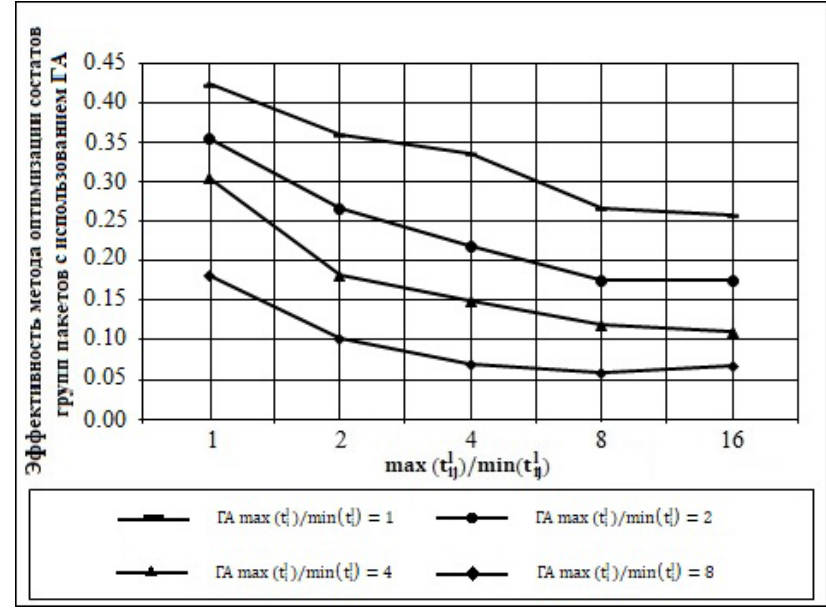

Рис. 2. Эффективность оптимизачии групп $\Pi Д\left(n=5, L=5, t^{z}=100, Z=2\right)$

[Fig. 2. Efficiency of optimizing groups of data packets $\left.\left(n=5, L=5, t^{z}=100, Z=2\right)\right]$

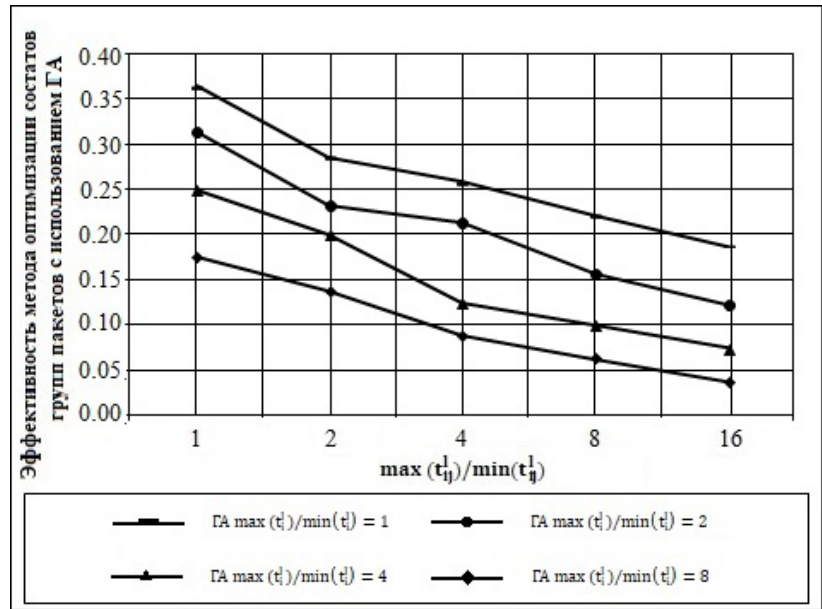

Рис. 3. Эффективность оптимизации групп $\Pi Д\left(n=5, L=5, t^{z}=100, Z=4\right)$

[Fig. 3. Efficiency of optimizing groups of data packets $\left.\left(n=5, L=5, t^{z}=100, Z=4\right)\right]$

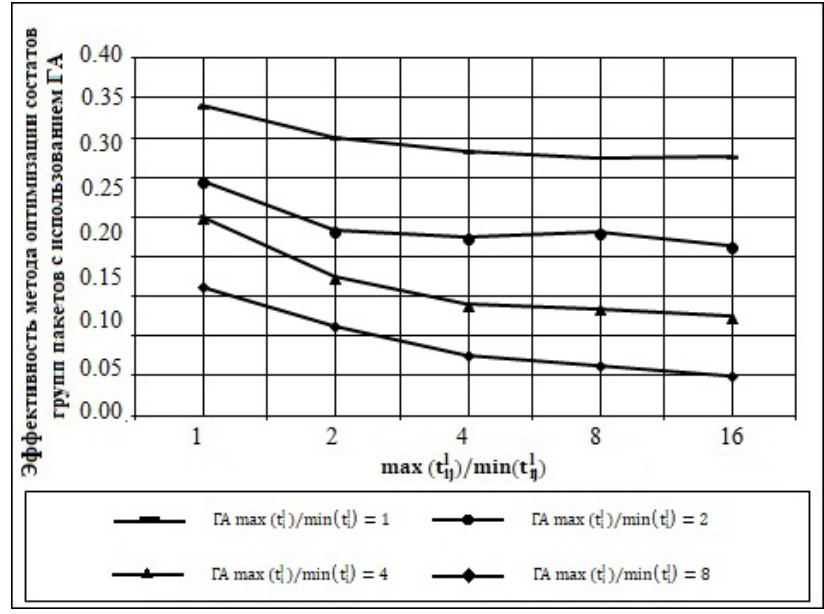

Рис. 4. Эфбективность оптимизации групп ПД $\left(n=5, L=5, t^{z}=200, Z=2\right)$

[Fig. 4. Efficiency of optimizing groups of data packets $\left.\left(n=5, L=5, t^{z}=200, Z=2\right)\right]$

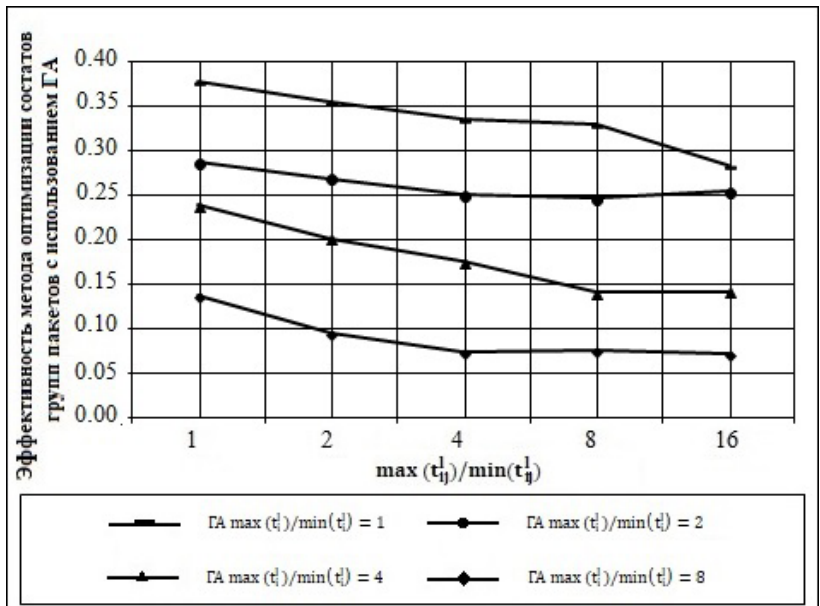

Рис. 5. Эффективность оптимизации групп $\Pi Д\left(n=5, L=5, t^{z}=200, Z=4\right)$

[Fig. 5. Efficiency of optimizing groups of data packets $\left.\left(n=5, L=5, t^{z}=200, Z=4\right)\right]$ 
Анализ результатов исследований при значениях при $n=5, L=5, t^{z}=100$ и $t^{z}=200$, $Z=2$ и $Z=4$, при изменении значений параметров $\max \left(t_{i j}^{l}\right) / \min \left(t_{i j}^{l}\right)$ и $\max \left(t_{i}^{l}\right) / \min \left(t_{i}^{l}\right)$ в указанных диапазонах показал, что максимальная эффективность применения метода оптимизации групп пакетов с использованием ГА фиксируется при значениях $t^{z}=100, Z=2$, при $\max \left(t_{i}^{l}\right) / \min \left(t_{i}^{l}\right)=1, \max \left(t_{i j}^{l}\right) / \min \left(t_{i j}^{l}\right)=1$ и составляет $42 \%$. Эффективность применения метода оптимизации составов групп ПД снижается при увеличении значений отношений $\max \left(t_{i}^{l}\right) / \min \left(t_{i}^{l}\right)$ и $\max \left(t_{i j}^{l}\right) / \min \left(t_{i j}^{l}\right)$. При этом увеличение неоднородности длительностей обработки данных разных типов на сегментах конвейера влияет на снижение эффективности применения метода более значительно, чем увеличение неоднородностей длительностей переналадок сегментов конвейера. Увеличение количества групп ПД (с $Z=2$ до $Z=4)$ при прочих равных значениях входных параметров приводит к снижению эффективности применения метода на $5 \%$ и более. Увеличение значений длительностей интервалов функционирования конвейерной системы с $t^{z}=100$ до $t^{z}=200$ также приводит к снижению эффективности применения метода оптимизации групп пакетов на 5-7 \%.

\section{ЗАКЛЮЧЕНИЕ}

Современное состояние теории расписаний характеризуется отсутствием методов, позволяющих выполнять планирование обработки ПД при задании ограничений на интервалы времени функционирования конвейерной системы. По этой причине разработан метод оптимизации составов групп ПД, обрабатываемых в течение заданных интервалов, использующий генетические алгоритмы. Сформулирован способ построения начального решения по составам групп ПД, которое используется при формировании начальной популяции. Сформулирован метод, основывающийся на генетических алгоритмах, позволяющий выполнять оптимизацию составов групп ПД, обрабатываемых в течение заданных интервалов времени. Его компонентами являются: способ кодирования решений по составам групп ПД в виде хромосом, способы реализации операторов генетических алгоритмов- мутации, скрещивания и селекции, позволяющие формировать новые решения по составам групп ПД. Рассмотренный метод оптимизации решений по составам групп $N^{z}(z=\overline{1, Z})$ предусматривает определение такого их состава, при котором простои сегментов в течение задаваемых интервалов времени функционирования конвейера являются минимальными. Реализация планирования обработки ПД с учетом ограничений с использованием рассмотренного метода позволяет до 45 \% уменьшить простои сегментов конвейера по сравнению с составами групп, не предусматривающими оптимизацию. Предложенный метод комплексного планирования обработки ПД с учетом ограничений на интервалы времени функционирования системы позволил устранить имеющийся пробел в современной теории расписаний.

Использование рассмотренного метода оптимизации составов групп пакетов возможно как при производственном планировании (формирование сменно-суточных заданий выпуска партий изделий (деталей) в конвейерной системе (задаваемые интервалы времени функционирования конвейера смены), так и при планировании обработки данных разных типов в случае, если вычислительные ресурсы являются выделяемыми во временное пользование за плату.

\section{КОНФЛИКТ ИНТЕРЕСОВ}

Авторы декларируют отсутствие явных и потенциальных конфликтов интересов, связанных с публикацией настоящей статьи.

\section{СПИСОК ЛИТЕРАТУРЫ}

1. Кротов, К. В. Комплексный метод определения эффективных решений по составам партий данных и расписаниям их обработки в конвейерных системах / К. В. Кротов // Вычислительные технологии. - Новосибирск: Из-во Института вычислительных технологий СО. - № 3. - 2018. - С. 58-76. DOI: 10.25743/ICT.2018.3.15984. 
2. Гермейер, Ю. Б. Игры с непротивоположными интересами / Ю. Б. Гермейер. - М. : Наука, 1976. - 327 с.

3. Mendez, C. A. State-of-the-art review of optimization methods for short-term scheduling ofbatch processes/C.A.Mendez//Computers and Chemical Engineering. - 2006. - V. 30. - P. $913-$ 946. DOI:10.1016/j.compchemeng.2006.02.008.

4. Ковалев, М. М. Модели и методы календарного планирования: Курс лекций / М. М. Ковалев. - Минск : Из-во БГУ, 2004. $63 \mathrm{c}$.

5. Tan, Y. Comparative Study of Different Approaches to Solve Batch Process Sheduling and Optimisation Problems / Y. Tan, W. Huangi, Y. Sun, Y. Yue // Proceedings of the 18th International Conference on Automation \& Computing. - Loughborough University. - Leicestershire. UK. - 2012. - P. 424-444.

6. Adonyi, $R$. Incorporating heat integration in batch process scheduling / R. Adonyi, J. Romero, L. Puigjaner, F. Friedler // Applied Thermal Engineering. - 2003. - V. 23. - P. 1743-1762. DOI: $10.1016 /$ S1359-4311(03)00141-8.

7. Kreipl, S. Planning and Scheduling in supply chains: An Overview of Issues in Practice / S. Kreipl // Production and Operations Management. - 2004. - V. 17. - No. 1. - P. 77-92.
8. Steiner, G. Minimizing the weight number of late jobs with Batch setup times and delivery costs on a single machine / G. Steiner, R. Zhang // Vienna: Itech Education and Published. - 2007. 436 p. DOI: $10.5772 / 5216$.

9. Koehler, F. Optimal Batch Schedules for Parrallel Machines / F. Koehler. S. Khuller // Algorithms and Data Structures: 13th International Symposium. - Berlin:Springer. - 2013. - P. 475486. DOI:10.1007/978-3-642-40104-6_41.

10. Yugma, G. C. Batching and Scheduling Algoritm for the diffusion Area in Semiconductor Manufacturing / G. C. Yugma, S. Dauzere-Peres, C. Artigues, O. Sibille // International Journal of Production Research. 2012. - V. 50. - no. 8. - P. 2118-2132. DOI: 10.1080/00207543.2011.575090.

11. Chandra, P. Managing Batch Processors to reduce lead time in a semiconductor packaging line / P. Chandra // International Jornal of Production Research. - 1997. - V. 35(3). - P. 611633.

12. Surjandari, I. The Batch Sheduling Model for Dynamic multiitem, Multilevel Production in an assembly Job-Shop with Parrallel Machines / I. Surjandari, A. Rachman, A. Dhini // International Journal of Technology. - 2015. - V. 1. - P. 8496. DOI: 10.14716/ijtech.v6i1.783.

Кротов Кирилл Викторович - канд. техн. наук, доцент, доцент кафедры информационных систем Института информационных технологий и управления в технических системах Севастопольского государственного университета.

E-mail: krotov_k1@mail.ru

ORCID iD: https://orcid.org/0000-0002-9670-6141 


\title{
APPLICATION OF GENETIC ALGORITHMS FOR THE DESIGN OF EFFECTIVE AND COMPREHENSIVE SCHEDULES FOR THE PROCESSING OF DATA PACKETS IN A PIPELINE SYSTEM AT SPECIFIC TIME INTERVALS
}

\author{
(c) 2020 K. V. Krotov ${ }^{凶}$ \\ Sevastopol State University \\ 33, Universitetskaya Str., 299053 Sevastopol, Russian Federation
}

\begin{abstract}
The article suggests a solution to the problem of designing effective data processing schedules with the processing pipeline functioning at specific time intervals. The problem of creating a comprehensive schedule is divided into three subtasks, each being solved at a certain level of the hierarchy. The first level determines the composition of the data packets. The second level determines the composition of groups of data packets, which are processed at specified time intervals. The third level determines the schedule of processing of the data packets included in each group. To solve the problem of creating comprehensive schedules within this hierarchy, the hierarchical game theoretic approach was applied. The article describes a hierarchical game model used to determine local optimal solutions for each level of the hierarchy. It also demonstrates that genetic algorithms can be used to determine the optimal composition of groups of data packets processed during specified time intervals in order to minimise the downtime of the pipeline segments. We propose a method to encode the decision making process regarding the composition of the data packets groups. The study determined the functioning of genetic operators which are used to search for local optimal solutions regarding the composition of the groups of packets based on the initial decision. We also developed software which optimises the composition of data packets and the composition of groups of data packets, and creates the processing schedule for the packets in the groups. The study demonstrated that the use of genetic algorithms in order to optimise the composition of groups of data packets allows for a $45 \%$ increase in the efficiency of using the time resource of the pipeline segments as compared to the initial solution.

Keyword: hierarchical game, time intervals of conveyer system functioning, groups of data packets, genetic algorithms.
\end{abstract}

\section{CONFLICT OF INTEREST}

The authors declare the absence of obvi-ous and potential conflicts of interest related to the publication of this article.

\section{REFERENCES}

1. Krotov K. V. A complex method for determining effective solutions for the composition of data batches and schedules of their processing in conveyor systems. Computational technologies, Publishing house Of the Institute of computational technologies SB RAS, Volume 23, No. 3, 2018. P. 58-76. DOI: 10.25743/ICT.2018.3.15984.

Krotov Kirill V. e-mail: krotov_k1@mail.ru
2. Hermeyer Yu. B. Games with non-contradictory interests. M. Nauka publishing House, 1976. $327 \mathrm{p}$.

3. Mendez C. A. et al. State-of-the-art review of optimization methods for short-term scheduling of batch processes. Computers and Chemical Engineering. 2006. V. 30. pp. 913-946. DOI:10.1016/j.compchemeng.2006.02.008

4. Kovalev M. M. Models and methods of calendar planning: a course of lectures. Minsk. Publishing house of BSU. 2004. 63 p.

5. Tan Y., Huangi W., Sun Y., Yue Y. Comparative Study of Different Approaches to Solve Batch Process Sheduling and Optimisation Problems. Proceedings of the 18th International Conference on Automation \& Computing. Loughborough University. Leicestershire. UK. 2012. P. 424-444. 


\section{К. В. Кротов}

6. Adonyi R., Romero J., Puigjaner L., Friedler F. Incorporating heat integration in batch process scheduling. Applied Thermal Engineering. 2003. V. 23. P. 1743-1762. DOI: 10.1016/ S1359-4311(03)00141-8.

7. Kreipl S. Planning and Scheduling in supply chains: An Overview of Issues in Practice. Production and Operations Management. 2004. V. 17. No. 1. P. 77-92.

8. Steiner G., Zhang R. Minimizing the weighted number of late jobs with Batch setup times and delivery costs on a single machine. Multiprocessor Scheduling, Theory and Applications. Edited by Levner E. Itech Education and Published. 2007. P. 85-98. DOI: 10.5772/5216.

9. Koehler F., Khuller S. Optimal Batch Schedules for Parrallel Machines. Algorithms and Data Structures: 13th International Symposium. Ber- lin. Springer. 2013. P. 475-486. DOI:10.1007/9783-642-40104-6_41.

10. Yugma G. C., Dauzere-Peres S., Artigues C., Sibille $O$. Batching and Scheduling Algoritm for the diffusion Area in Semiconductor Manufacturing. International Journal of Production Research. 2012. V. 50. No. 8. P. 2118-2132. DOI: 10.1080/00207543.2011.575090.

11. Chandra P. Managing Batch Processors to reduce lead time in a semiconductor packaging line. International Journal of Production Research. 1997. V. 35(3). P. 611-633.

12. Surjandari I., Rachman A., Dhini A. The Batch Scheduling Model for Dynamic multi-item, Multilevel Production in an assembly Job-Shop with Parrallel Machines. International Journal of Technology. 2015. V. 6. No 1. P. 84-96. DOI: $10.14716 /$ ijtech.v6i1.783.

Krotov Kirill V. - PhD in Technical Sciences, Associate Professor, Institute of Information Technology and Systems and Control Engineering, Sevastopol State University.

E-mail: krotov_k1@mail.ru

ORCID iD: https://orcid.org/0000-0002-9670-6141 